\title{
1 Title: Parental care influences social immunity in burying beetle larvae
}

3 Authors: C.E. Reavey, L. Beare \& S.C. Cotter

4 Address: School of Biological Sciences, Queen's University Belfast, MBC, 97 Lisburn Rd, 5 Belfast, BT9 7BL, UK

6 Corresponding author: Miss Catherine Reavey, School of Biological Sciences, Queen's

7 University Belfast, MBC, 97 Lisburn Rd, Belfast, BT9 7BL, UK. Tel: +44(0)2890972030,

8 Fax: +44(0)28 9097 5877. Email: creavey01@ qub.ac.uk

9 Keywords: antibacterial, ecological immunology, insect, lysozyme, Nicrophorus, parental 10 care, social immunity

11 Running title: Social immunity in burying beetle larvae 


\section{Abstract}

14 1. We provide evidence for social immunity in offspring of a sub-social species, the burying beetle, Nicrophorus vespilloides.

2. $N$. vespilloides is a carrion breeder, and in a similar fashion to the adult beetles, the offspring produce exudates that exhibit lytic activity, which are used to coat the breeding resource. This strategy defends against the microbial community.

19 3. The lytic activity in larval exudates declines as the brood develops, perhaps being most beneficial at the start of the breeding bout.

4. Changing levels of parental care through widowing/orphaning affects lytic activity in the larval exudates, with levels decreasing in the absence of both parents.

\section{Introduction}

The burying beetle, $N$. vespilloides (Figure S1, Supplementary Material), breeds on the carcass of small mammals and exhibits bi-parental care, one component of which is a social immune response (Cotter \& Kilner 2010b). Cotter \& Kilner suggest that "any type of immune response that has been selected to increase the fitness of the challenged individual and one or more recipients should be classified as social immunity" (2010b). The beetles coat the carcass with antimicrobial anal exudates (Cotter \& Kilner 2010a) in order to

30 minimise competition from the microbial community, delaying decomposition of the carcass

31 (Rozen et al. 2008). This social immune response is costly; antibacterial activity is only upregulated in the presence of a carcass (Cotter \& Kilner 2010a) and forced upregulation reduces lifetime reproductive success (Cotter et al. 2010). In N. vespilloides, both sexes

34 produce exudates, however, the females' exudates show higher levels of antibacterial activity 
than the males (Cotter \& Kilner 2010a). The beetles can also flexibly adjust the level of antibacterial activity in response to mate loss.

Observations have shown that the larvae also produce exudates throughout their development, though their potential antibacterial activity is unknown. With production of these exudates being so costly to the parents, it would benefit them if their offspring were also able to partake in carcass preservation. Furthermore, we know that larvae of this species can survive without parental care, albeit at a reduced rate and with a resulting poorer quality (Eggert et al. 1998). Their ability to survive the loss of both parents may be due in part to the production of antibacterial substances. Here we ask whether 1) antibacterial activity is present, 2) how it changes during larval development and 3) whether larvae, like adults, can flexibly alter levels of antibacterial activity in response to changing conditions, for example, 46 presence/absence of parents.

\section{$47 \quad$ Materials and Methods}

\section{N. vespilloides colony}

The colony was established from a pedigreed $N$. vespilloides colony at the

Department of Zoology, University of Cambridge. Beetles were maintained as described

51 previously (Cotter \& Kilner 2010a).

\section{Experiment 1 - characterising antibacterial activity across the larval stage}

Exudates were collected from all larvae in a brood using a capillary tube and pooled in a single eppendorf tube. This enabled us to use a known volume of exudate in the later analysis $(1 \mu \mathrm{l})$. Larvae were sampled from day 1 (hatching) to day 5 (dispersal). Day 1 and 2 larvae were so small that exudate collection with a capillary tube was not possible. Instead, the tip of the abdomen was gently pressed against a single punched circle of filter paper for 
every larva in the brood. Whilst volume was impossible to control for in these samples, we collected $<1 \mu \mathrm{l}$ of exudate sample on each filter paper circle, and so the level of lytic activity would be a slight under-estimate compared to day 3,4 and 5 larvae. All samples were stored at $-20^{\circ} \mathrm{C}$ until testing. Larvae were sampled from fifty-five families, though some were sampled on more than one day, giving 75 samples in total. Forty-two families were sampled from once, nine families were sampled from twice and five families were sampled from three times. The numbers of larvae from which samples were collected and pooled on each day were as follows: day $1=10.33$ larvae $+/-2.01$, day $2=14.63$ larvae $+/-1.49$, day $3=9.65$ larvae $+/-1.24$, day $4=14.27$ larvae $+/-1.88$ and day $5=7.17$ larvae $+/-2.29$.

\section{Experiment 2 - the effect of reduced parental care on larval antibacterial activity}

Sixty pairs were established and were assigned to one of four treatment groups, male removed, female removed, both removed, or neither removed (control). On the day of hatching, parents were removed according to the treatment and the larval exudate sampled daily as described above. Exudate collection was more successful for this experiment, sixty families were sampled daily for five days giving 300 samples in total. Breeding success was recorded for all families.

\section{Antibacterial activity}

Lysozyme-like antibacterial activity was measured as described previously (Cotter \&

Kilner 2010a). In brief, $1 \mu \mathrm{l}$ of each sample was pipetted into a hole in an agar plate inoculated with freeze-dried Micrococcus lysodeikticus cells and incubated for 24 hours at $33^{\circ} \mathrm{C}$. We selected Micrococcus lysodeikticus as it is a soil bacterium, which is the breeding environment of the burying beetle. It is one of the microorganisms analysed in Hall et al. (2011) and in that study was inhibited by secretions from Nicrophorus. It is also the main

81 bacteria used for lytic plates in studies on burying beetles (Cotter et al. 2010, Steiger et al. 2011, Arce et al. 2012) and many other insect species e.g. locusts (Wilson et al. 2002). Filter 
paper circles were placed directly onto the surface of the agar. Clear zones in the agar were measured with digital calipers and calibrated against a lysozyme standard (Figure S2,

Supplementary Material). In order to test for the potential mechanical differences between using filter paper and punched holes, we compared samples tested using both methods and found no difference between the size of the clear zone produced by $1 \mu$ of sample pipetted into a hole and that produced using filter paper placed on the surface of the agar $\left(\mathrm{F}_{1,25}=0.27\right.$, $\mathrm{P}=0.61)$. We were therefore able to reliably compare samples measured using the different techniques. Whilst other methods are now available to measure antimicrobial activity, the majority of analyses to date in this and many other insect species have been carried out using zones of inhibition on agar plates. Using this method allows us to compare across studies to some extent.

\section{Statistical analyses}

Antibacterial activity was log-transformed to approximate normality. Lytic data from both experiments was analysed with Restricted Estimate Maximum Likelihood (REML) models in Genstat 15 (VSN International, Hemel Hempstead, UK) with family included as a random effect to account for multiple testing from each family. Breeding data were analysed using a generalised linear model (GLM). Carcass weight and interaction terms were included

100 in all models but were removed due to non-significance. Figures were produced in R 2.15.1

101 (Development Core Team, 2013).

\section{Results and Discussion}

We show for the first time in this study that the larval exudates contain antibacterial

104 substances. It has been shown previously that insect larvae have the ability to produce antibacterial secretions e.g. blowfly larvae, L. sericata (Kerridge et al. 2005), however this 
107 hatched larvae and declined throughout the brood (REML: $\mathrm{F}_{1,49}=51.27, \mathrm{P}<0.001$; Figure 1).

108 Indeed, as the exudate volume collected from day 1 and day 2 larvae was $<1 \mu$, the lytic

109 activity at this stage is actually slightly underestimated, and so in reality should be even

110 higher than observed. If the trend had been in the other direction i.e. larval exudate activity

111 increasing with age, we could not have drawn reliable conclusions as in this case a lower

112 activity in day 1 and day 2 larvae could have been due to a smaller exudate volume. As we

113 did not collect a fixed amount of exudate from day 1 and 2 larvae (although volume $<1 \mu \mathrm{l}$ ),

114 we tested whether activity was dependent on the number of larvae from which it was

115 collected, however there was no effect $\left(\mathrm{REML}: \mathrm{F}_{1,27}=0.51, \mathrm{P}=0.48\right)$. The pattern of decline

116 in larval lytic activity mirrors that of the parents; highest when larvae arrive on the carcass

117 (Cotter et al. 2013). The need for lytic exudates may fall after a high amount of initial

118 preservation, resulting in increasing sterility. For the parents, assistance from the larvae may

119 mean that they do not need to invest as heavily in lytic activity, which could promote further

120 reproductive success and longevity.

With regards parental removal experiments, preliminary data exploration showed that the effects of removing males and females were very similar but different from the other two treatment groups. Therefore we grouped these two treatments into a single treatment representing "one parent removed". Lytic activity was much lower in larvae where both parents were removed (REML: $\mathrm{F}_{2,46}=3.66, \mathrm{P}=0.033$; Figure $2 \mathrm{a}$ ) and there was a significant effect of brood age, with the pattern being very similar to experiment 1 (REML: $\mathrm{F}_{1,210}=$

127 119.95, P < 0.001). These results illustrate that, like the parents (Cotter \& Kilner 2010a), the larvae exhibit plasticity with regards lytic activity levels. Whilst we hypothesised that this antibacterial activity may contribute to their survival in orphaned conditions, activity actually

130 decreased. This drop may be due to the fact that the larvae must now invest in other

131 activities, for example self-feeding, at the detriment to lytic investment. Their condition is 
132 also likely to be compromised even at this stage, which may cause a decline in lytic activity.

133 A different experimental set-up may be required to unveil an upregulation of lytic activity in

134 the larvae, for example using a rotten carcass but maintaining parental assistance i.e. a greater

135 requirement for combatting microorganisms but without the strain of self-feeding. The

136 number of larvae produced was lower when both parents were removed post-hatching (GLM:

$137 \mathrm{~F}_{2,51}=4.30, \mathrm{P}=0.019$; Figure $2 \mathrm{~b}$ ), but the mean weight of larvae was not affected (GLM:

$138 \mathrm{~F}_{2,49}=2.07, \mathrm{P}=0.137$ ). This is consistent with findings from previous studies (Rozen et al.

139 2008; Arce et al. 2012).

$140 \quad$ Future experiments should consider the cost of lytic activity from the larval

141 perspective. The larvae may be constrained developmentally if the mechanism for

142 production in the parents also drives production in the larval stage. In light of plasticity from

143 parents and their offspring, future studies should consider the scope for conflict. For

144 example, if the larvae didn't produce antibacterial exudates, would the parents be forced to

145 produce more? To conclude, we show that larvae produce antibacterial exudates and that

146 parental care influences the extent of social immunity.

\section{Acknowledgements}

148 CER was supported by a DEL studentship, SCC was supported by a NERC

149 fellowship (NE/H014225/2). Author contributions: SCC co-designed the experiments,

150 analysed the data, and co-wrote the paper; LB co-designed the experiments and collected the

151 data; CER co-designed the experiments, collected the data and co-wrote the paper. 


\section{References}

154 Arce, A. N., Johnston, P. R., Smiseth, P. T. \& Rozen, D. E. 2012. Mechanisms and fitness

155 effects of antibacterial defences in a carrion beetle. Journal of Evolutionary Biology, 25, 930-

156937.

157 Cotter, S. C. \& Kilner, R. M. 2010a. Sexual division of antibacterial resource defence in

158 breeding burying beetles, Nicrophorus vespilloides. Journal of Animal Ecology, 79, 35-43.

159 Cotter, S. \& Kilner, R. 2010b. Personal immunity versus social immunity. Behavioral

160 Ecology, 21, 663-668.

161 Cotter, S., Topham, E., Price, A. \& Kilner, R. 2010. Fitness costs associated with mounting a

162 social immune response. Ecology Letters, 13, 1114-1123.

163 Cotter, S. C., Littlefair, J. E., Grantham, P. J. \& Kilner, R. M. 2013. A direct physiological

164 trade-off between personal and social immunity. Journal of Animal Ecology, 84, 846-853.

165 Eggert, A. K., Reinking, M. \& Müller, J. K. 1998. Parental care improves offspring survival 166 and growth in burying beetles. Animal Behaviour, 55, 97-107.

167 Hall, C.L. et al. (2011) Inhibition of Microorganisms on a Carrion Breeding Resource: The

168 Antimicrobial Peptide Activity of Burying Beetle (Coleoptera: Silphidae) Oral and Anal

169 Secretions. Environmental Entomology, 40, 669-678.

170 Kerridge, A., Lappin-Scott, H. \& Stevens, J. 2005. Antibacterial properties of larval

171 secretions of the blowfly, Lucilia sericata. Medical and veterinary entomology, 19, 333-337. 
172 Rozen, D. E., Engelmoer, D. J. P. \& Smiseth, P. T. 2008. Antimicrobial strategies in burying

173 beetles breeding on carrion. Proceedings of the National Academy of Sciences, 105, 17890-

17417895.

175 Steiger, S. et al. (2011) Sex differences in immunity and rapid upregulation of immune

176 defence during parental care in the burying beetle, Nicrophorus orbicollis. Functional

177 Ecology, 25, 1368-1378.

178 Wilson, K. et al. (2002) Coping with crowds: Density-dependent disease resistance in desert

179 locusts. PNAS, 99, 5471-5475.

180

181

182

183 Figure legends

184 Figure 1

185 The decline in the antibacterial activity of larval exudate over time. Larvae hatch on 186 day 1 and disperse from the carcass on day 5.

188 Figure 2

189

The effect of removing one or both parents on a) the antibacterial activity of larval

190 exudate and b) the number of larvae dispersing from the carcass. Means and SEs in a) are

191 predicted values from a REML model controlling for family. 
$192 \quad$ Figures

$193 \quad$ Figure 1

194

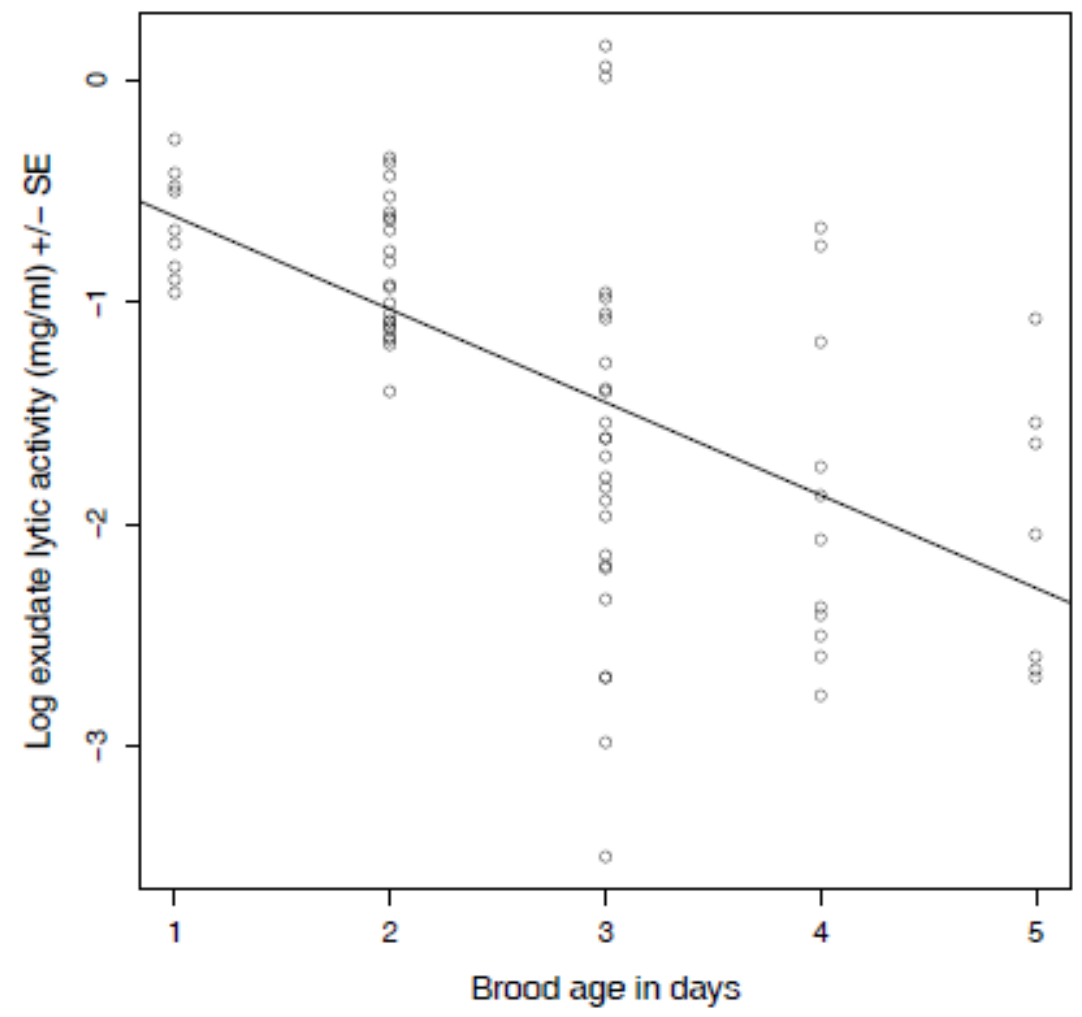

195

196 
$197 \quad$ Figure 2

198

199

a)

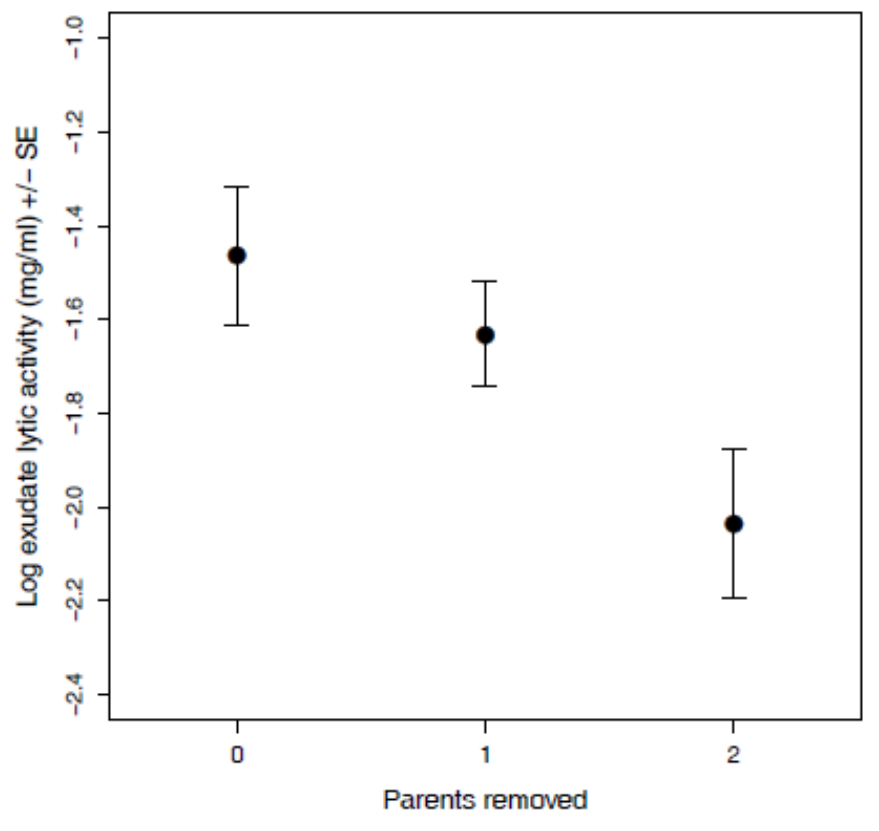

201

b)

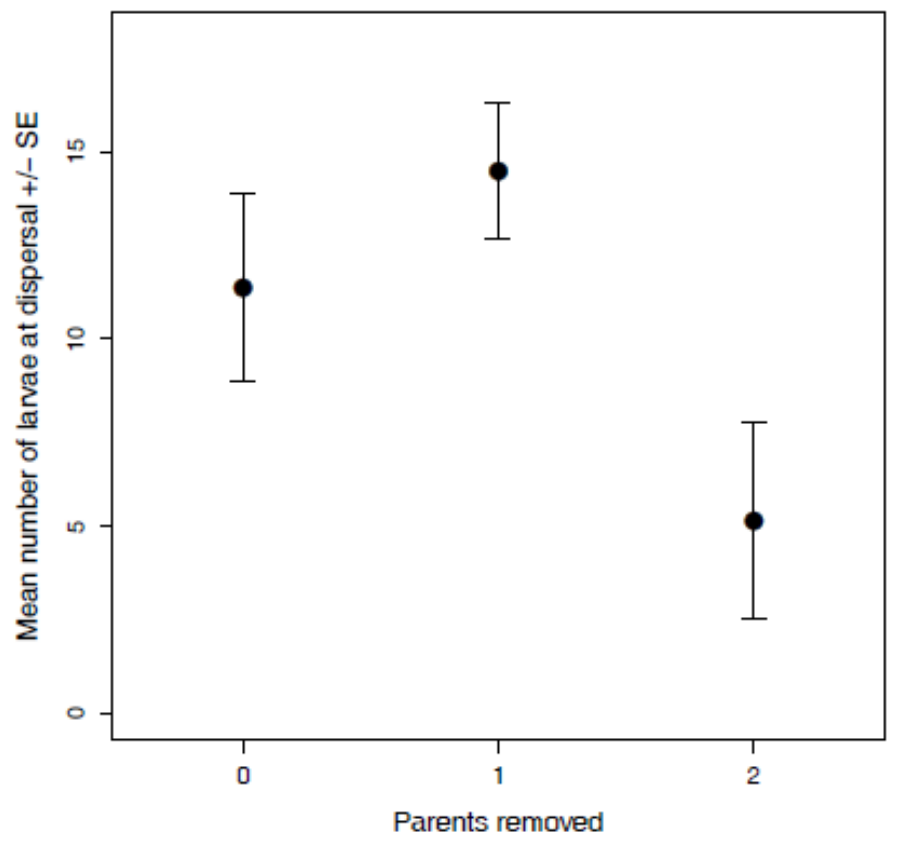

